\title{
Capsule Commentary on Marc et al., Reliability and Validity of the Haitian Creole PHQ-9
}

\author{
Kurt Kroenke, $M D^{1,2}$ \\ 'VA HSR\&D Center for Health Information and Communication; Regenstrief Institute, Inc, Indianapolis, IN, USA; ${ }^{2}$ Indiana University School of \\ Medicine, Indianapolis, IN, USA.
}

J Gen Intern Med 29(12): 1691

DOI: $10.1007 / \mathrm{s} 11606-014-2998-3$

(c) Society of General Internal Medicine 2014

$\mathrm{T}$ his study by Marc et al. ${ }^{1}$ developed and tested a HaitianCreole translation of the PHQ-9 in men who have sex with men (MSM) living in the Republic of Haiti. The investigators demonstrated unidimensionality of the translated PHQ-9, moderately high internal consistency reliability ( $\alpha=0.78$ ), preliminary evidence of construct validity (i.e., greater depression in HIV-positive subjects), and no evidence of differential item functioning (DIF) across age, education, sexual orientation or HIV status.

The PHQ-9 has become one of the most widely-used depression measures due to its brevity, inclusion of the nine criteria for major depressive disorder (MDD), utility as a screening, severity, and outcome measure, and public domain status. $^{2}$ The PHQ-9 has been translated into more than 80 languages (many available at www.phqscreeners.com). Because it is difficult to obtain funding solely for translations, new translations of a measure are often developed as part of studies funded for other reasons (e.g., epidemiological research, multi-national pharmaceutical trials, or by investigators with an interest in conditions assessed principally by patient-report). Also, when a measure has already been widely translated, a publication validating a new translation has added value when it provides data on a new population (e.g., MSM) or "higher-level" psychometric analyses (e.g., factor analysis and DIF). Most studies examining the factor structure of the PHQ-9 have found it to be unidimensional; thus, even though MDD comprises several types of symptoms (e.g., somatic, affective, cognitive), it appears the MDD syndrome is captured by a single overarching construct. One implication is that the clinician need not decide if somatic symptoms are due to a comorbid medical disease or depression, but can use an inclusive approach to counting all symptoms towards a diagnosis of MDD. ${ }^{3}$ Fewer studies have examined DIF for the PHQ-9, and these have typically (but not invariably) shown that the PHQ-9 items perform similarly across most demographic and disease subgroups. Patient-reported outcome measures like the PHQ family of scales and the NIH PROMIS measures ${ }^{4}$ www.nihpromis.org) are becoming increasingly important, ${ }^{5}$ and valid translations are necessary for evaluating and improving global health.

Conflict of Interest: The author has no conflicts of interest with the material in this article.

Corresponding Author: Kurt Kroenke, MD; VA HSRD Center for Health Information and Communication; Regenstrief Institute, Inc, Indianapolis, IN, USA (e-mail: kkroenke@regenstrief.org).

\section{REFERENCES}

1. Marc LG, Henderson WR, Desrosiers A, Testa MA, Jean SE, Akom EE. Reliability and validity of the Haitian Creole PHQ-9. J Gen Intern Med 2014; (Article SPI \#2951)

2. Kroenke K, Spitzer RL, Williams JBW, Löwe B. The Patient Health Questionnaire somatic, anxiety, and depressive symptom scales: a systematic review. Gen Hosp Psychiatry. 2010;32:345-359.1.

3. Simon GE, Von Korff M. Medical co-morbidity and validity of DSM-IV depression criteria. Psychol Med. 2006;36:27-36.

4. Cella D, Riley W, Stone A, Rothrock N, Reeve B, Yount S, et al. The Patient-Reported Outcomes Measurement Information System (PROMIS) developed and tested its first wave of adult self-reported health outcome item banks: 2005-2008. J Clin Epidemiol. 2010;63:1179-94.

5. Glasgow RE, Riley WT. Pragmatic measures: what they are and why we need them. Am J Prev Med. 2013;45:237-43. 